\title{
Radio Propagation Characteristics in the Large City
}

\author{
YoungKeun Yoon*, JongHo Kim, MyoungWon Jung, and YoungJun Chong \\ *Radio Technology Research Department, ETRI, Republic of Korea \\ ykyoon@etri.re.kr, jonghkim@etri.re.kr, mwjung@etri.re.kr, and yjchong@etri.re.kr
}

\begin{abstract}
This paper describes various radio propagation characteristics in the large city such as Seoul in Republic of Korea and talks on the closed form of a received interfering signal intensity to a victim system for the coexistence with two different systems. Actually, it is difficult to how to choose the optimum radio propagation model for predicting on the interference impact because of various environment conditions or system limits, even if the theoretic radio propagation models are known for various services. Specially, it is not known for the available median path loss model in order to calculate the interfering signal intensity to a victim system between the fixed communication link and mobile communication system for none line of site environment. Therefore, we measured the radio propagation characteristics in the large city and discussed with the adequate median path loss and shadowing characteristics in this paper
\end{abstract}

Keywords - radio, propagation, path loss, measurement, FM

\section{INTRODUCTION}

Long term evolution (LTE) to be enable operators to better and more cost effectively transport the rapidly growing volume of mobile data traffic has been developing. Also, the fixed microwave link system is required to transport the audio or video broadcasting data. FM broadcasting repeater is a sort of a microwave link system. In terms of the spectrum at $1.7 \mathrm{GHz}$ bands, LTE and FM broadcasting repeater's carrier frequency channels are in the adjacent bands each other in Korea. Therefore, we might predict the potential interference impacts in the coexistence cases. We should choose the radio propagation model in the various environments like urban, suburban, and open area.

Generally, the extended Hata model is used to none line of sight as well as line of sight. And, free space model, of which ITU-R P.525, is applied for line of sight between a transmitter and a receiver on the communication system [1]. Also, this median path loss model and shadowing with log-normal distribution can calculate the cell coverage corresponding to the sensitivity level of a receiver. It is very useful of predicting the channel characteristic without the complex measurement procedure. Currently, the choice of the median path loss model to calculate the link loss is so simple. For example, operators choose the extended Hata model for predicting the site coverage in the macro cell or TV broadcasters does the ITU-R P.1546 model with time varying and spatial rates [2]. However, this choice may be happened the large error and inaccuracy for the interfering link calculation between two different services in the large city (e.g. Fixed link and mobile communication link). Because one mobile communication system use the urban case of extend
Hata model, in the other hands, other fixed microwave system may use the free space model or ITU-R P. 1546 model due to different environment conditions or system limits like antenna height.

In this paper, we try to solve this analysis problem of different two systems with different median path loss model. Therefore, we measured the radio propagation characteristics at the $1.7 \mathrm{GHz}$ bands and calculated the received signal strength in the adjacent channel to predict the impact on LTE system from FM broadcasting repeater interference in the large city.

Finally, we found to use the median path loss corresponding to various environments in the large city. Measurement results are good mapping with the simulation results with different path loss model, respectively.

\section{SySTEM PARAMETERS}

Let me show the considered fixed services. They are a sort of FM broadcasting repeater system such as microwave link at $1.7 \mathrm{GHz}$ operation bands. But, FM broadcasting repeater is different from the microwave link in terms of the location of site, except for a fixed service. FM broadcasting repeater's transmitter is the top of building in the city, but, the microwave link's one is on the top of mountain.

In this paper, two fixed services operating at $1.7 \mathrm{GHz}$ bands in Korea are described. One is the fixed FM broadcasting repeater running by Far East Broadcasting Company (FEBC) system and the other is a system by Seoul Broadcasting Station (SBS) system. Fixed broadcasting repeater system consists of a kind of a studio-transmitter link (STL) system [3]-[5]. A STL sends a radio station's or television station's audio and video from the broadcast studio to a radio transmitter or television transmitter in another location. This is often necessary because the best locations for an antenna are on top of a mountain, where a much shorter tower is required, but where a studio is completely impractical. Even in flat regions, the center of the station's allowed coverage area may not be near the studio location or within a populated area where a transmitter would be frowned upon by the community, so the antenna must be placed several kilometres away. Depending on the locations that must be connected, a station may choose either a point to point (PTP) link on another special radio frequency, or a newer all-digital wired link via a dedicated T1 or E1 (or larger-capacity) line. Radio links can also be digital, or the older analogue type, or a hybrid of the two. Even on older all-analogue systems, multiple audio and data channels can be sent using subcarriers. Stations that 
employ an STL usually also have a transmitter-studio link (or TSL) to return telemetry information. Both the STL and TSL are considered broadcast auxiliary services (BAS).

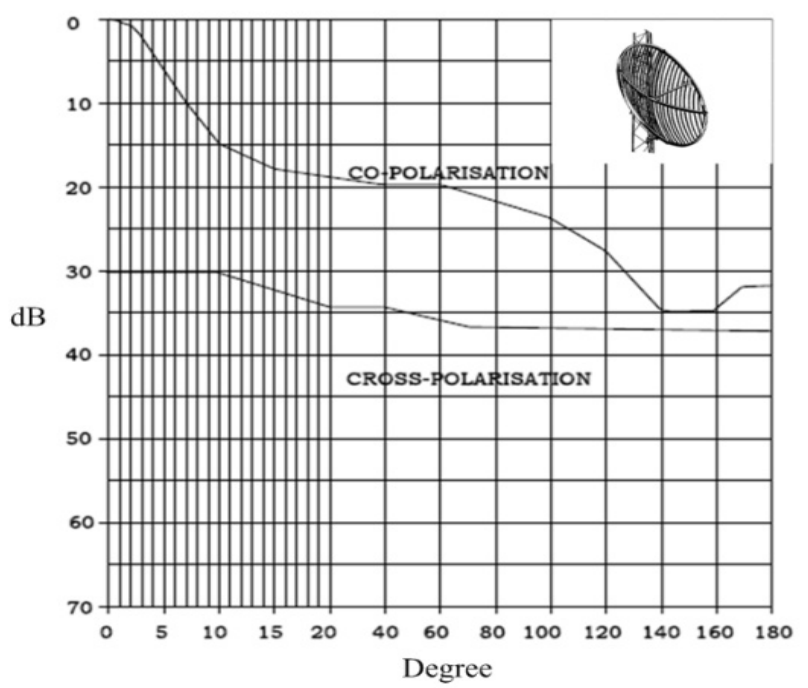

Figure 1. FM broadcasting repeater's antenna elevation pattern (e.g. Grid parabola)

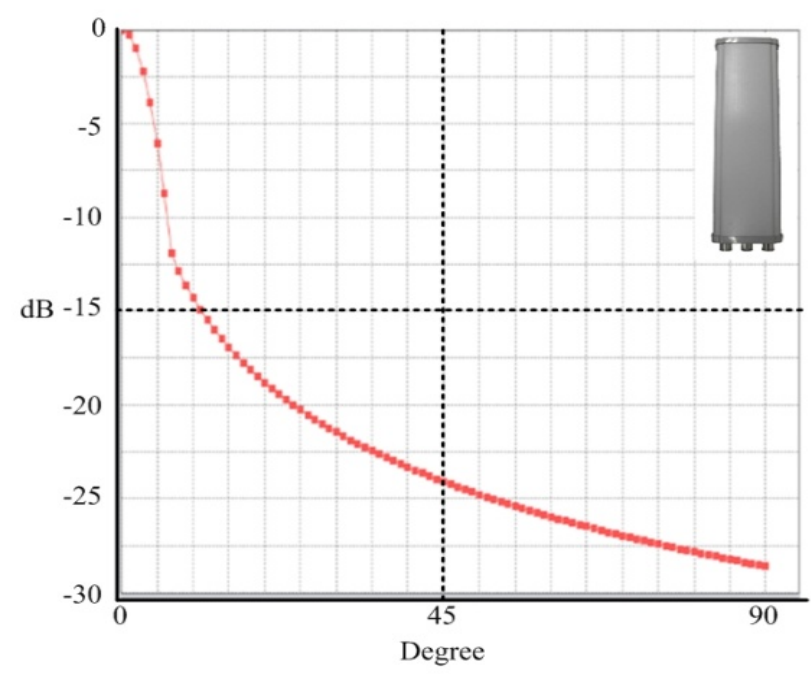

Figure 2. LTE base station's antenna elevation pattern (e.g. Linear)

FEBC's station consists of a kind of analogue STL system of TFT8300 [4]. It has a transmit power of 5.0Watts and the channel bandwidth of $230 \mathrm{kHz}$. Antenna type operating at $1.7 \mathrm{GHz}$ bands is the grid parabola and has the gain of $26 \mathrm{dBi}$ including $2 \mathrm{~dB}$ feeder loss with 6.5 degree elevation beamwidth shown in Figure 1. The antenna height of a transmit station is about $35 \mathrm{~m}$. The antenna height of a receiving station is about $625 \mathrm{~m}$ in Gwanaksan Mt. SBS's station consists of a kind of digital STL system of SL9003Q [5]. It has a transmit power of 1.0 Watts and the channel bandwidth of $230 \mathrm{kHz}$. Antenna type operating at $1.7 \mathrm{GHz}$ bands is the grid parabola and has the gain of $26 \mathrm{dBi}$ including $2 \mathrm{~dB}$ feeder loss with 6.5 degree elevation beamwidth shown in Figure 1. The antenna height of a transmit station is about $70 \mathrm{~m}$. The antenna height of a receiving station is about $625 \mathrm{~m}$ in Gwanaksan Mt.

The LTE specification provides downlink peak rates of 100 $\mathrm{Mbit} / \mathrm{s}$, uplink peak rates of $50 \mathrm{Mbit} / \mathrm{s}$ and QoS provisions permitting a transfer latency of less than $5 \mathrm{~ms}$ in the radio access network. LTE has the ability to manage fast-moving mobiles and supports multi-cast and broadcast streams. LTE supports scalable carrier channel bandwidths, from $1.4 \mathrm{MHz}$ to $20 \mathrm{MHz}$ and supports both frequency division duplexing (FDD) [6]. In this paper, LTE is considered for supporting carrier channel bandwidths of $5 \mathrm{MHz}$ and is assumed that LTE is reverse link in FDD at adjacent channel of FM broadcasting repeater bands at $1.7 \mathrm{GHz}$. Its antenna type operating at $1.7 \mathrm{GHz}$ bands is the linear x-pol antenna and has the gain of $15 \mathrm{dBi}$ including $3 \mathrm{~dB}$ feeder loss with 7.0degree elevation beamwidth shown in Figure 2. The height of a receive antenna is about $10 \mathrm{~m}$. An antenna beam's down tilt angle is -3 degree.

\section{III.MEASUREMENTS}

\section{A. Measurement Paths}

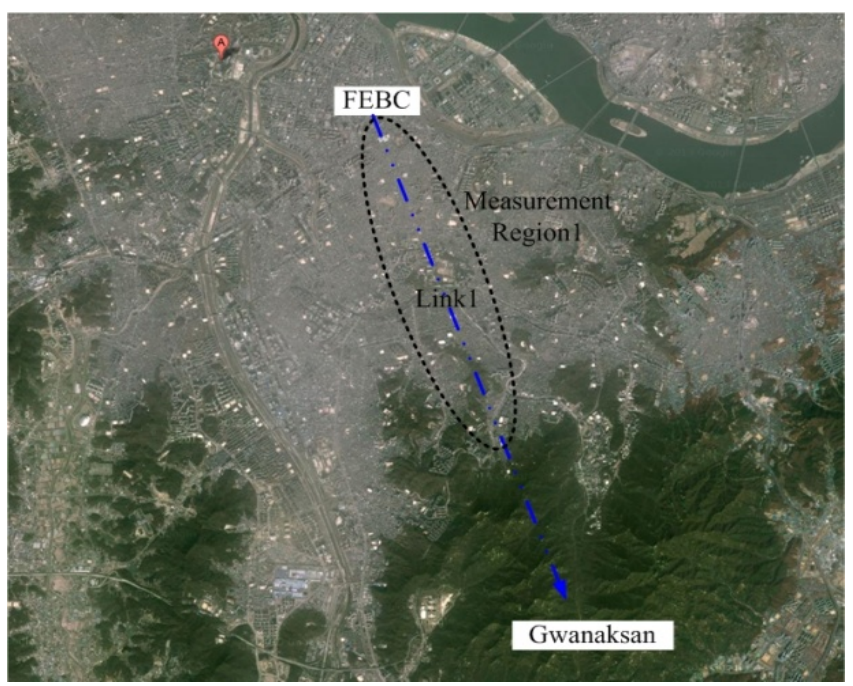

Figure 3. Measurement route (e.g. FEBC to Gwanaksan Mt.)

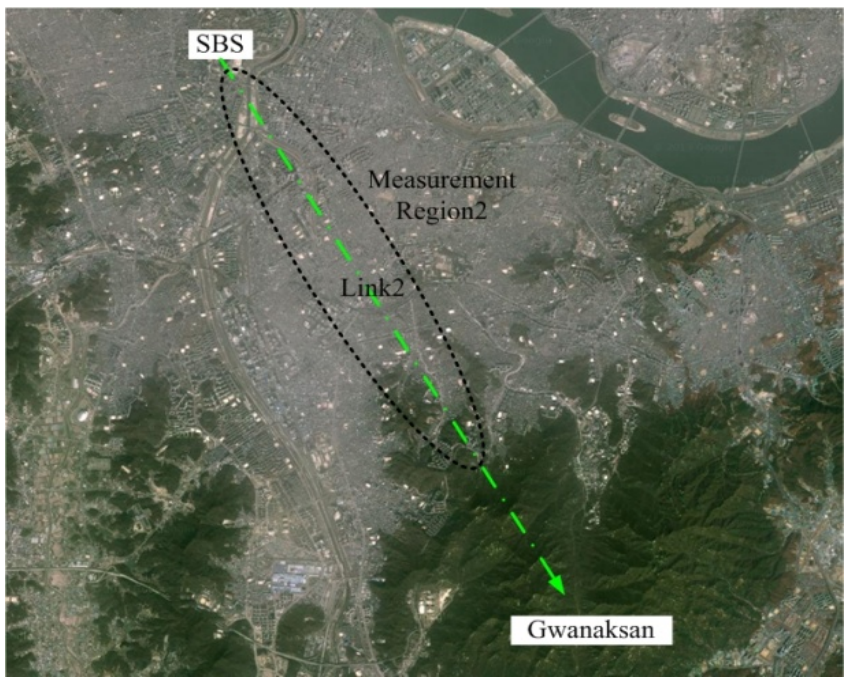

Figure 4. Measurement route (e.g. SBS to Gwanaksan Mt.) 
Figure 3 and Figure 4 show each measurement path from the broadcasting station to the front of Gwanaksan Mt.

Link 1 in the Figure 3 means the measurement path from the FEBC broadcasting station to FEBC repeater in Gwanaksan Mt. Link1 distance between a broadcasting station and a receiving antenna of FEBC site on the Gwanaksan Mt. is about $10 \mathrm{~km}$. Link 2 in the Figure 4 means the measurement path from the SBS broadcasting station to SBS repeater in Gwanaksan Mt. Link2 distance between a broadcasting station and a receiving antenna of SBS site on the Gwanaksan Mt. is about $12.4 \mathrm{~km}$.

\section{B. Measurement Environment}

Figure 5 shows the measurement method in order to measure the radio propagation characteristic such as median path loss and shadowing. Measurement was performed on the road with a moving vehicular in the large city with huge or small buildings. Vehicular moves from a FM broadcasting station (Transmitter) to the front of Gwanaksan Mt. Here, the transmit antenna height of FM broadcasting station is $\mathrm{H}_{1}$ and the receiving antenna height of $\mathrm{FM}$ broadcasting repeater on the top of Gwanaksan Mt. is $\mathrm{H}_{2}$. The receiving antenna height of measurement vehicular is $h_{v}$. The antenna height $\mathrm{H}_{1}$ of FEBC or SBS station is $35 \mathrm{~m}$ or $70 \mathrm{~m}$, respectively. The antenna height $\mathrm{H}_{2}$ of $\mathrm{FM}$ broadcasting repeater on the top of Gwanaksan Mt. is $625 \mathrm{~m}$. The antenna height $h_{v}$ of a vehicular is $2.5 \mathrm{~m}$.

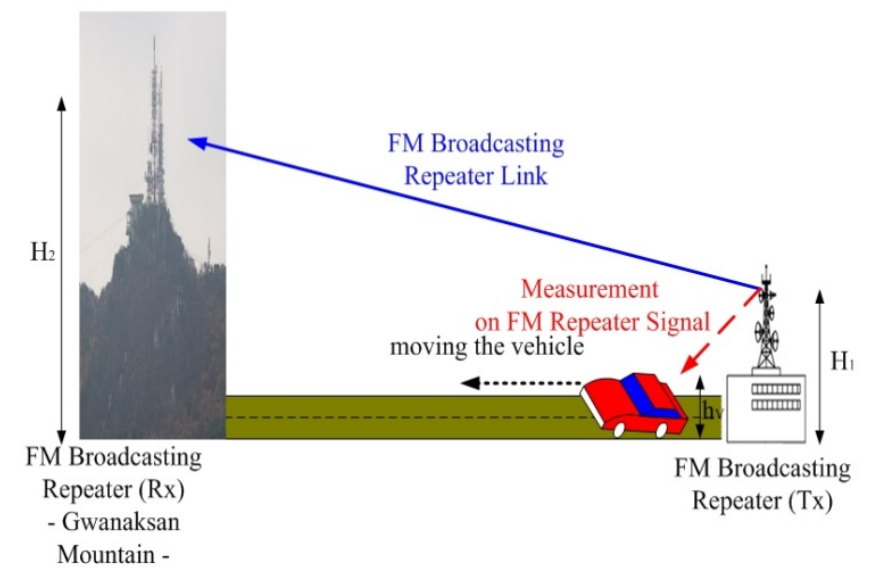

Figure 5. Measurement method

\section{IV.RESULTS \& ANALYSIS}

\section{A. Theoretic Median Path loss Models}

For comparing the measurement results and theoretic analysis, we looked for candidate median path loss model. [1]

For line of sight in the large city, we used the free space model

$$
\mathrm{PL}_{\mathrm{FSL}}(\mathrm{dB})=32.44+10 \log \left\{\left(\frac{\mathrm{H}_{1}-\mathrm{h}_{\mathrm{v}}}{1000}\right)^{2}+\mathrm{d}^{2}\right\}+20 \log (\mathrm{f})
$$

This model describes the theoretical minimum propagation path loss achievable in free space conditions. It is appropriate to use on paths were unobstructed direct line of sight propagation could be expected. They are example for point to point fixed service links such as FM broadcasting microwave link or links over short distance in open area, and so on.

For non-line of sight of urban, suburban, and rural communication link applications at $1.7 \mathrm{GHz}$ operation bands, we used the extended Hata model, which was developed and found by a European study committee (COST231). The basic formula for the median propagation loss in $\mathrm{dB}$ given by the extended Hata propagation loss model is as follows: The available range of parameters for which this model is considered, are valid to the operating frequency of less than $3 \mathrm{GHz}$ and the receive antenna height of 1 to $10 \mathrm{~m}$.

$$
\begin{aligned}
& \text { In case of urban environment, } \\
& \mathrm{PL}_{\mathrm{Urban}}(\mathrm{dB})= \\
& \quad 46.3+33.9 \log (\mathrm{f})-13.82 \log \left(\max \left\{30, \mathrm{H}_{1}\right\}\right)+ \\
& {\left[44.9-6.55 \log \left(\max \left\{30, \mathrm{H}_{1}\right\}\right)\right] \log (\mathrm{d})-\mathrm{a}\left(\mathrm{h}_{\mathrm{v}}\right)-\mathrm{b}\left(\mathrm{H}_{1}\right)}
\end{aligned}
$$

In case of suburban environment,

$$
\begin{aligned}
& P L_{\text {Suburban }}(\mathrm{dB})=\mathrm{PL}_{\mathrm{Urban}}- \\
& \quad 2\{\log [(\min \{\max \{150, \mathrm{f}\}, 2000\}) / 28]\}^{2}-5.4
\end{aligned}
$$

In case of rural environment,

$$
\begin{aligned}
& P_{\text {Rural }}(\mathrm{dB})= \\
& \mathrm{PL}_{\text {Urban }}-4.78\{\log [\min \{\max \{150, \mathrm{f}\}, 2000\}]\}^{2} \\
& \quad+18.33 \log [\min \{\max \{150, \mathrm{f}\}, 2000\}]-40.94
\end{aligned}
$$

where,

$$
\begin{aligned}
\mathrm{a}\left(\mathrm{h}_{\mathrm{v}}\right)= & (1 \cdot 1 \log (\mathrm{f})-0.7) \min \left\{10, \mathrm{~h}_{\mathrm{v}}\right\}- \\
& (1.56 \log (\mathrm{f})-0.8)+\max \left\{0,20 \log \left(\frac{\mathrm{h}_{\mathrm{v}}}{10}\right)\right\} \\
\mathrm{b}\left(\mathrm{H}_{1}\right)= & \min \left\{0,20 \log \left(\frac{\mathrm{H}_{1}}{30}\right)\right\}
\end{aligned}
$$

Long term fading calling for the shadowing has a different standard deviation according to a measurement environments: the standard deviation to both the separation distance range from $0.1 \mathrm{~km}$ to $0.2 \mathrm{~km}$ between a transmitter and a receiver and the below roof is $17 \mathrm{~dB}$. The standard deviation to the separation distance range from $0.2 \mathrm{~km}$ to $0.6 \mathrm{~km}$ is $\sigma=17$ $20(\mathrm{~d}-0.2) \mathrm{dB}$. And, the standard deviation to the separation distance range larger than $0.6 \mathrm{~km}$ is $9 \mathrm{~dB}[1]$.

\section{B. Results Comparison}

Figure 6 and Figure 7 show the received signal strength intensity to a meausrement vehicular with both omin direction antenna and its height of $2.5 \mathrm{~m}$. As shown in Figure 3 and Figure 4, each measurement path from the broadcasting station to the front of Gwanaksan Mt. is different. Of course, it is sure that RSSI has different value through vehicular moving path. Figure 6 and Figure 7 are median path loss including the shadowing from both measurement and analysis. The fluctation of a shadowing is about $6 \sim 10 \mathrm{~dB}$ in the FEBC shown in Figure 6 to Gwanaksan path and $10 \sim 13 \mathrm{~dB}$ in the SBS shown in Fiugre 7 to Gwanaksan path. This shadowing value is larger approximately $3 \sim 4 \mathrm{~dB}$ than a standard deviatoin 
of shadowing given by COST231 [1]. Also, the median path loss of the measurement is good mapping with theoretic extended Hata model including the combined urban case and suburban case, except for the measurement point of the near location from FM broadcasting station as a transmitter. Results in Figure 6 and Figure 7 show that the median path loss model could use the urban case of extended Hata model by shorter than about $15 \mathrm{~km}$ from a FM broadcasting station and could use suburban or open area case of extended Hata model from longer than $15 \mathrm{~km}$ in case of the receiving antenna heigth of $2.5 \mathrm{~m}$ in the large city in Korea at $1.7 \mathrm{GHz}$ bands.

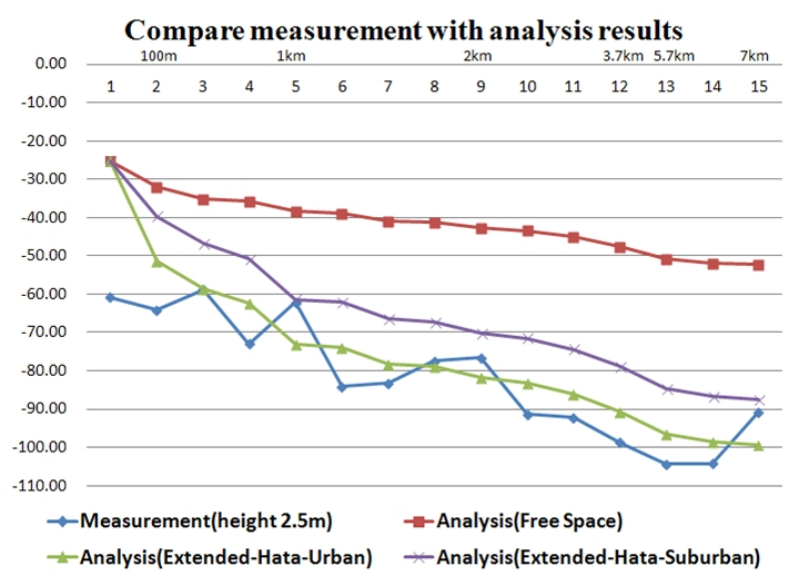

Figure 6. FEBC's RSSI in the large city

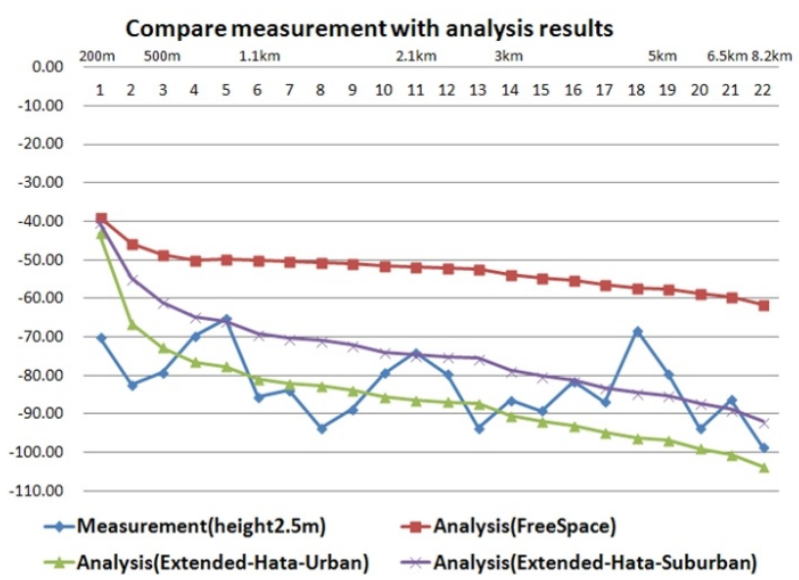

Figure 7. SBS's RSSI in the large city

\section{Interference calculation}

We found to apply for different median path loss model and realistic shadowing standard deviation in the large city in Korea according to the relative distance between a fixed FM broadcasting station and a mobile communication receiver such as LTE base station. In addition, for predicting on LTE interference impact in the adjacent channel, we used both the RSSI given to the measurement at FM broadcasting repeater's carrier frequency in shown in Figure 6 and Figure 7 and the correction value of the LTE antenna height of $10 \mathrm{~m}$ instead of the vehicular antenna height of $2.5 \mathrm{~m}$. Finally, RSSI to LTE base station is derived as follow: Here, RSSI means totally received interfering signal strength intensity due to out of band emission of FM broadcasting repeater. FM broadcasting repeater's emission means FM broadcasting repeater's out of band interfering signal transmitting into LTE base station in related with the interfering link as shown in Figure 8. Figure 9 and Figure 10 show FEBC Station's emission and SBS's emission characteristics given to a realistic measurement.

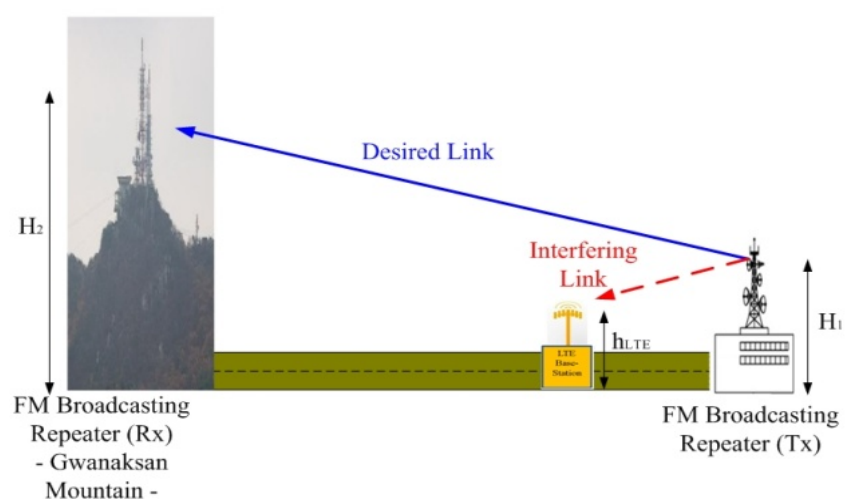

Figure 8. Desired and interfered link

$$
R S S I(\mathrm{dBm})=I_{\mathrm{ooB}, \Delta \mathrm{F}}+G_{\mathrm{Rx}}\left(H_{1}, h_{\mathrm{LTE}}, \delta\right)+\mathrm{a}\left(h_{\mathrm{LTE}}\right)
$$

where, $R S S I$ depicts totally received interfering signal strength intensity, $I_{\mathrm{OoB}, \Delta \mathrm{F}}$ means a theoretic received interfering signal strength intensity to the LTE base station using out of band emission(ooB) characteristics in shown Figure 9 and Figure 10 to the separation frequency $(\Delta \mathrm{F})$ from offset frequency of FM station channel band edge with channel bandwidth of $5 \mathrm{MHz}$ derived from the measured FM station's signal intensity. $G_{\mathrm{Rx}}\left(H_{1}, h_{\mathrm{LTE}}, \delta\right)$ depicts the LTE antenna gain considering the direction elevation angle from main lobe to LTE base station. This antenna gain includes the antenna height $\left(H_{1}\right)$ of FM broadcasting station, the antenna height $h_{\text {LTE }}$ of LTE base station, and down tilt angle of LTE base station antenna. This down tilt angle is assumed as -3 degree. $\mathrm{a}\left(h_{\mathrm{LTE}}\right)$ means the correction factor of the antenna height converting the vehicular antenna height of $2.5 \mathrm{~m}$ to LTE base station antenna height of $10 \mathrm{~m}$.

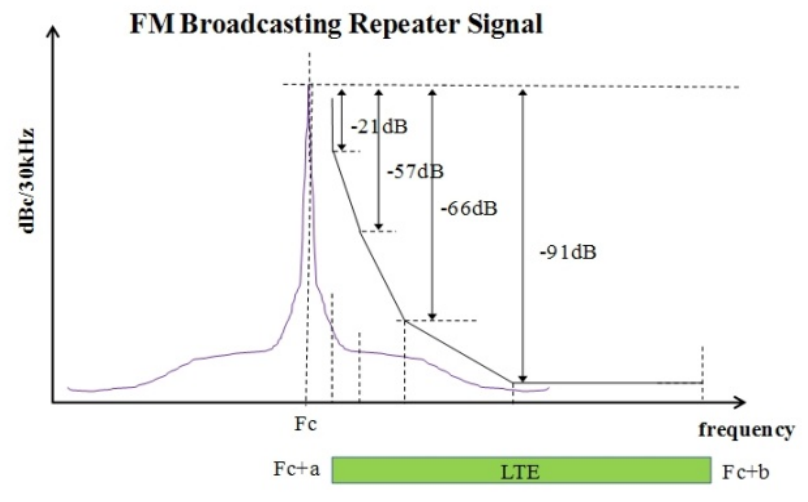

Figure 9. FEBC Station's Emission Characteristics 


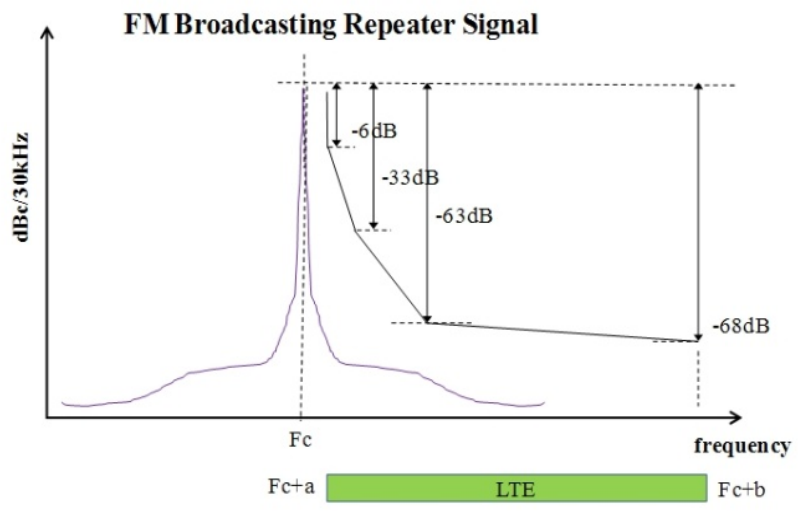

Figure 10. SBS Station's Emission Characteristics

For calculating the interference impact, a permissible interference level of $-108 \mathrm{dBm} / 5 \mathrm{MHz}$ to LTE base station should be satisfied. This value is derived from the protection ratio of LTE base station to prevent from FM broadcasting repeater's interference. This protection ratio $(=\mathrm{I} / \mathrm{N})$ depicts the interfering signal to the noise level of the victim system [7][8]. This value of $\mathrm{I} / \mathrm{N}$ to LTE base station is $-6 \mathrm{~dB}$. Finally, the separation distance of $2 \mathrm{~km}$ from FM broadcasting station to LTE base station should be required in order to satisfy the permissible interfering level of $-108 \mathrm{dBm} / 5 \mathrm{MHz}$ to LTE base station.

\section{Conclusions}

This paper described the required median path loss characteristics and long term fading in the large city based on the measurement results. Also, we found that the median path loss of the measurement in the large city is good mapping with theoretic extended Hata model including combined the urban model with suburban model. In addition, for the protection of LTE base station from FM broadcasting repeater's interference, the separation distance from a fixed interferer to a victim receiver was given in none line of sites. For the coexistence between a fixed station such as FM broadcasting station and a mobile communication system like LTE, it is expected to use these results. Also, antenna correction factor is very important to predict the radio propagation with the accuracy and is applied to calculate the interference in the adjacent channel bands.

\section{ACKNOWLEDGMENT}

This work was supported by the IT R\&D program of $\mathrm{MSIP} / \mathrm{KCA}$

\section{REFERENCES}

[1] ERC Report 68, Monte-Carlo Simulation Methodology for the Use in Sharing and Compatibility Studies between Different Radio Services or System, CEPT, June 2002

[2] ITU-R, Method for point-to-area predictions for terrestrial services in the frequency range $30 \mathrm{MHz}$ to $3000 \mathrm{MHz}$, REC. ITU-R P. 1546, Oct. 2009

[3] FCC, CFR Title 47: Telecommunication Chapter I-Federal Communications Commission Subchapter C-Part 73-Broadcast Radio Services

[4] http://www.tftinc.com/stlproducts.html

[5] http://www.moseleysb.com/mb/starlink_family.html

[6] Motorola, Long Term Evolution (LTE): A Technical Overview, Technical White Paper, 2007

[7] ECC Report 131, Derivation of a Block Edge Mask (BEM) for Terminal Stations in the 2.6GHz Frequency Bands $(2055-2690 \mathrm{MHz})$, CEPT, Feb. 2009

[8] Yongsup Shim, Ilkyoo Lee, and Seungkeun Park, "The impact of LTE UE on Audio Devices," ETRI Journal, vol.35, no.2, Apr. 2013, pp.332335

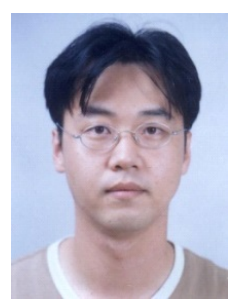

environments
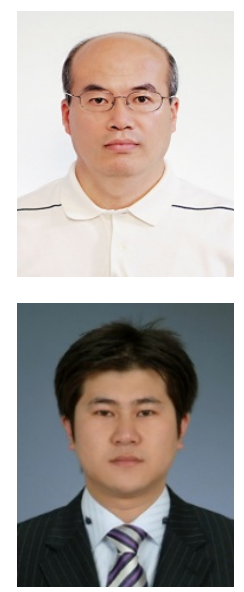

outdoor environments.

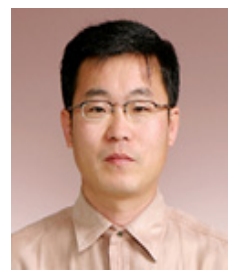
Department. He is currently involved in the development of the digital ultranarrow band Walky-Talky. His research interests include RF circuit and systems

YoungKeun Yoon was born in Chungbuk, Korea. He received the B.E. and M.E. degrees in radio engineering from National Chungbuk University, Korea in 1997, 1999, respectively. Since 2000, he has been worked in Electronics and Telecommunications Research Institute (ETRI). He has been involved in the research of radio resource management and propagation since 2003. His main interests are radio propagation study for mobile communication and spectrum engineering study in indoor and outdoor

JongHo Kim received his $\mathrm{BS}$, $\mathrm{MS}$, and $\mathrm{PhD}$ in electronic engineering from Chungnam National University, Daejeon, Rep. of Korea, in 1986, 1988, and 2006, respectively. Since 1989, he has been working for ETRI, Daejeon, Rep. of Korea, where he is a principal member of the engineering staff of the Radio Technology Department. His main interests are radio propagation and spectrum engineering.

MyoungWon Jung received B.S. and M.S. degree in Electronic Engineering from Chungnam National University, Daejeon, Korea, in 2006, 2008. Since 2009 he has been working for Electronics and Telecommunications Research Institute (ETRI) where he is a senior member of research staff of the Radio Technology Department. His main interests are radio propagation study for mobile communication and millimeter wave propagation study in indoor and

YoungJun Chong received the B.S. degree from the Jeju University, Jeju island, Korea, in 1992, and the M.S. degree in electronics engineering in 1994 from Sogang University. And Ph.D degree in Electronic Engineering from Chungnam National University, Daejeon, Korea, in 2005 respectively. Since 1994 he has been with ETRI, Dasjeon, Korea, where he is a leader of spectrum engineering section principl member of the research staff of the Radio Technology 